\title{
CONVERGENCE TOWARDS SELF-SIMILAR ASYMPTOTIC BEHAVIOR FOR THE DISSIPATIVE QUASI-GEOSTROPHIC EQUATIONS
}

\author{
JOSÉ A. CARRILLO \\ ICREA (Institució Catalana de Recerca i Estudis Avançats) \\ and Departament de Matemàtiques, Universitat Autònoma de Barcelona \\ E-08193 Bellaterra, Spain \\ E-mail: carrillo@mat.uab.es \\ LUCAS C. F. FERREIRA \\ Departament de Matemàtiques, Universitat Autònoma de Barcelona \\ E-08193 Bellaterra, Spain \\ E-mail: lcff@mat.uab.es
}

\begin{abstract}
This work proves the convergence in $L^{1}\left(\mathbb{R}^{2}\right)$ towards an Oseen vortex-like solution to the dissipative quasi-geostrophic equations for several sets of initial data with suitable decay at infinity. The relative entropy method applies in a direct way for solving this question in the case of signed initial data and the difficulty lies in showing the existence of unique global solutions for the class of initial data for which all properties needed in the entropy approach are met. However, the estimates obtained for the constructed global solutions in $L^{1} \cap L^{2}$ show the asymptotic simplification of the solutions even for unsigned initial data emphasizing the character of this equation to behave linearly for large times.
\end{abstract}

1. Introduction. In this work, we deal with the asymptotic behavior for a system of equations arising in two dimensional models of fluid mechanics, the so called quasigeostrophic equations. Atmospheric fronts were modelled in [9] by these equations without dissipation. The dissipative two dimensional quasi-geostrophic (2DQG) equations are derived from general quasi-geostrophic equations [25] in the special case of constant potential vorticity and constant buoyancy frequency [9] and they read as follows

2000 Mathematics Subject Classification: 35Q, 35B40.

Key words and phrases: quasi-geostrophic equations, asymptotics of solutions, self-similarity. The paper is in final form and no version of it will be published elsewhere. 


$$
\begin{cases}\frac{\partial \theta}{\partial t}-\kappa \Delta^{\gamma} \theta+(u \cdot \nabla) \theta=0, & x \in \mathbb{R}^{2}, t>0 \\ \theta(x, 0)=\theta_{0}(x), & x \in \mathbb{R}^{2},\end{cases}
$$

with $\kappa>0$ and $\gamma \in[0,1]$. The velocity field $u=\left(u_{1}, u_{2}\right)$ is divergence free, $\nabla \cdot u=0$, and determined from the potential temperature $\theta$ through the stream function $\psi=(-\Delta)^{-\frac{1}{2}} \theta$ by

$$
u=\left(-R_{2} \theta, R_{1} \theta\right)=\left(-\partial_{2} \psi, \partial_{1} \psi\right)=\nabla^{\perp} \psi .
$$

In other words, the velocity field is given in terms of Riesz transforms of the potential temperature that will be denoted by the operator $u=R[\theta]$.

Let us remark the existence of a special self-similar solution of the 2DQG. Fix $\kappa=1$ for the remainder of the paper and let $\gamma \in[0,1]$. Let $G_{\gamma}(t, x) \in L^{2}\left(\mathbb{R}^{2}\right)$ be defined as $\widehat{G_{\gamma}}(t, \xi)=e^{-t|\xi|^{2 \gamma}}$ for all $t>0$. Note that the stream function $\psi_{G_{\gamma}}=(-\Delta)^{-\frac{1}{2}}\left(G_{\gamma}\right)$, associated with the function $G_{\gamma}$, is radial. Indeed, this follows from the Fourier transform expression of $\psi_{G_{\gamma}}$

$$
\widehat{\psi_{G_{\gamma}}}(t, \xi)=\frac{1}{|\xi|} e^{-t|\xi|^{2 \gamma}}
$$

and the fact that Fourier transform preserves radial symmetry. Using the expression of the velocity field (1.2) and the fact that both $\psi_{G_{\gamma}}$ and $G_{\gamma}$ are radial functions, we have the following orthogonality property $(u \cdot \nabla) G_{\gamma}=\nabla^{\perp} \psi_{G_{\gamma}} \cdot \nabla G_{\gamma}=0$. Since $G_{\gamma}(t, x)$ is the fundamental solution of the operator $\frac{\partial}{\partial t}-\Delta^{\gamma}$, then it takes the delta Dirac distribution $\delta_{0}$ as initial data weakly as measures. In other words, $\theta(t, x)=G_{\gamma}(t, x)$ with its corresponding stream function is a source-type solution for the 2DQG equations.

We will show that this particular self-similar solution of $2 \mathrm{DQG}$ for $\gamma=1$, i.e. the heat kernel $G=G_{1}$ for the equation

$$
\begin{cases}\frac{\partial \theta}{\partial t}-\Delta \theta+u \nabla \theta=0, & x \in \mathbb{R}^{2}, t>0, \\ \theta(x, 0)=\theta_{0}(x), & x \in \mathbb{R}^{2},\end{cases}
$$

plays the same role as the Oseen vortex for the two dimensional Navier-Stokes (2DNSV) equations in vorticity-velocity formulation. The Oseen vortex has recently been proved by T. Gallay and C.E. Wayne $[15,16]$ to be globally asymptotically stable for all initial integrable vorticities regardless of their sign. Moreover, they obtain decay rates towards the Oseen vortex under suitable additional conditions on the initial data. The space $L^{1}\left(\mathbb{R}^{2}\right)$ is natural for the 2DNSV due to the homogeneity of the Biot-Savart law while for the $2 \mathrm{DQG}$ the right homogeneity space is $L^{2}\left(\mathbb{R}^{2}\right)$. However, the "mass" is preserved, at least formally, for (1.3).

Existence of self-similar solutions with local basins of attraction has been shown in [4] in Lorentz spaces and some spaces of tempered distributions with the right homogeneity, i.e. $\theta_{0} \in L^{2, \infty}\left(\mathbb{R}^{2}\right)$. Assuming that the initial data belongs to the strong $L^{p}$ space with the right homogeneity $\theta_{0} \in L^{2}\left(\mathbb{R}^{2}\right)$, it was proved in [4] that there exist global solutions for small initial data satisfying the decay

$$
\lim _{t \rightarrow \infty}\|\theta(t)\|_{L^{2}\left(\mathbb{R}^{2}\right)}=0 .
$$


The main result of this paper shows that the self-similar solution $G$ characterizes the long-time asymptotics of (1.1) with $\gamma=1$ for signed initial data in $L^{2}(m) \subset L^{1}\left(\mathbb{R}^{2}\right)$ with $m>1$, see section 3 for a precise definition of the weighted space $L^{2}(m)$. More precisely, we prove that given $\theta_{0} \in L_{+}^{2}(m)$, there exists a unique global in time positive solution $\theta(t, x)$ with

$$
\|\theta(t, x)-\eta G(t, x)\|_{L^{1}\left(\mathbb{R}^{2}\right)} \leq C t^{-1 / 2},
$$

where $\eta$ is identified by the initial mass

$$
\eta=\int_{\mathbb{R}^{2}} \theta_{0}(x) d x,
$$

and the constant $C$ is explicitly computable in terms of the initial data. This asymptotic simplification result is also true for unsigned initial data in $L^{2}(m)$ and in $L^{1} \cap L^{2}\left(\mathbb{R}^{2}\right)$ with different rates and no explicit constants, see last section for precise statements.

The result for signed initial data is achieved following some of the key ideas of [15], namely, the existence of an important Liapunov functional for the 2DNSV equations: the relative entropy. The use of relative entropy arguments in the case of signed initial data has been very successful in coping with similar situations in diffusion-dominated nonlinear equations $[14,6,3]$ for which the convective terms are asymptotically negligible. In fact, the entropy dissipation arguments for positive initial data, used in [15] and in our case, are similar to the basic ideas applied by G. Toscani $[30,31]$ for the linear heat equation based on the logarithmic Sobolev inequality [18]. Finally, let us point out that the method used cannot be extended to the cases $0<\gamma<1$ since we do not know how to find a suitable relative entropy functional.

However, the new difficulty that we face for unsigned initial data is that the homogeneity of the equation in $L^{2}\left(\mathbb{R}^{2}\right)$ and not in $L^{1}\left(\mathbb{R}^{2}\right)$ produces a nonautonomous dynamical system in self-similar variables. This new feature avoids the possibility of LaSalle-type arguments and thus, we cannot describe the long time asymptotics for unsigned initial data as in [15]. Here, we proceed by direct estimates showing the decay to zero of the bilinear terms in suitable norms. This procedure succeeds but it does not give the optimal rates expected from the signed case.

On the other hand, let us mention that although global existence and properties of the solutions of the dissipative QG equations in different functional settings have been analyzed in $[10,8,12,19,32,33,34,27,28,4]$, no one of these results contain a wellposedness theory of solutions in the class $L^{1}\left(\mathbb{R}^{2}\right) \cap L^{2}\left(\mathbb{R}^{2}\right)$ with all the properties needed to use entropy methods. Some properties regarding the time decay of solutions and their derivatives in this class have been obtained in [11] and [28]. Therefore, we will devote next section to show the global existence, uniqueness, $L^{p}$ estimates on the solutions and their derivatives and maximum principle for initial data in the class $L^{1}\left(\mathbb{R}^{2}\right) \cap L^{2}\left(\mathbb{R}^{2}\right)$. Moreover, after self-similar change of variables, we will also need to show the decay at infinity of solutions, i.e., they belong to $L^{2}(m)$ and their regularity to be able to apply entropy-like arguments. This will be the objective of Section 3. Finally, we will show in Section 4 the main results of this work concerning the asymptotic simplification of this model in the sets of initial data mentioned above. 
2. Global existence of smooth solutions in $L^{2}\left(\mathbb{R}^{2}\right)$. Let us start this section by recalling some known facts about the heat kernel in $L^{p}\left(\mathbb{R}^{2}\right)$ whose norm will be denoted by $\|\cdot\|_{p}$. Let $G(t)$ be the convolution operator with the heat kernel $G(x, t)$, i.e., $G(t) f=$ $G(\cdot, t) * f$ where

$$
G(x, t)=\frac{1}{4 \pi t} e^{-\frac{|x|^{2}}{4 t}}
$$

The standard scaling invariances of the heat kernel $G(x, t)$ such as $G(x, t)=t^{-1} G\left(x t^{-\frac{1}{2}}, 1\right)$ and $\left(\nabla_{x}^{k} G\right)(x, t)=t^{-1-\frac{|k|}{2}}\left(\nabla_{x} G\right)\left(x t^{-\frac{1}{2}}, 1\right)$ imply the following consequence:

Lemma 2.1. Let $1 \leq r \leq p \leq \infty$ and $k \in \mathbb{N}$. Given $\theta_{0} \in L^{r}\left(\mathbb{R}^{2}\right)$, then

$$
\left\|\nabla_{x}^{k} G(t) \theta_{0}\right\|_{p} \leq C t^{-\frac{|k|}{2}-\frac{1}{r}+\frac{1}{p}}\left\|\theta_{0}\right\|_{r}
$$

Moreover, given $\theta_{0} \in L^{2}\left(\mathbb{R}^{2}\right)$ and $2<q \leq \infty$, then

$$
\sup _{0<t<\infty} t^{\frac{\alpha}{2}}\left\|G(t) \theta_{0}\right\|_{q} \leq\left\|\theta_{0}\right\|_{2} \quad \text { and } \quad \lim _{t \rightarrow 0^{+}} t^{\frac{\alpha}{2}}\left\|G(t) \theta_{0}\right\|_{q}=0
$$

where $\alpha=1-\frac{2}{q}$.

Proof. The first part of lemma is a well-known property of heat kernel. In order to prove the second part, we use (2.1) with $r=2$ and $p=q$ to obtain

$$
\left\|G(t) \theta_{0}\right\|_{q} \leq C t^{-\left(\frac{1}{2}-\frac{1}{q}\right)}\left\|\theta_{0}\right\|_{2}=C t^{-\frac{\alpha}{2}}\left\|\theta_{0}\right\|_{2}
$$

Now, let us take an approximating sequence $\left\{\theta_{0, m}\right\} \subset L^{2} \cap L^{q}\left(\mathbb{R}^{2}\right)$ such that $\theta_{0, m} \rightarrow \theta_{0}$ in $L^{2}\left(\mathbb{R}^{2}\right)$. Using again (2.1) with $r=p=q$, we get $\left\|G(t) \theta_{0, m}\right\|_{q} \leq C\left\|\theta_{0, m}\right\|_{q}$ and thus

$$
\lim _{t \rightarrow 0^{+}} t^{\frac{\alpha}{2}}\left\|G(t) \theta_{0, m}\right\|_{q}=0
$$

for all $m \in \mathbb{N}$. Previous estimate (2.3) implies that the sequence $t^{\frac{\alpha}{2}} G(t) \theta_{0, m}$ belongs to $B C\left((0, \infty) ; L^{q}\left(\mathbb{R}^{2}\right)\right)$ and converges in $\|\cdot\|_{q}$ to the function $t^{\frac{\alpha}{2}} G(t) \theta_{0}$ uniformly in any time interval $(0, T)$ for all $T>0$. Here, we have denoted by $B C$ the class of bounded and continuous functions from the corresponding interval into a Banach space. Therefore, we conclude taking into account (2.4).

The main aim of this section is to show the global well-posedness and the regularity of the 2DQG in the homogeneity space $L^{2}\left(\mathbb{R}^{2}\right)$. Furthermore, we will obtain estimates on the time decay of $L^{p}$-norms of the solution and its spatial derivatives. Let us introduce suitable function spaces to analyze the Cauchy problem for the system (1.3) based on $L^{p}$-spaces.

Definition 2.2. Let $E_{q, T}$ be the space of all functions $h(t, x)$, with $t \geq 0$ and $x \in \mathbb{R}^{2}$, such that

$$
h(t, x) \in B C\left([0, T), L^{2}\right) \quad \text { and } \quad t^{\frac{\alpha}{2}} h(t, x) \in B C\left([0, T), L^{q}\right)
$$

where $\alpha=1-\frac{2}{q}$. The norm in $E_{q}$ is naturally defined by

$$
\|h\|_{E_{q}}=\sup _{0<t<T} t^{\frac{\alpha}{2}}\|h(t, \cdot)\|_{q}+\sup _{0<t<T}\|h(t, \cdot)\|_{2} .
$$


Let us make precise the notion of mild solution we deal with:

Definition 2.3. A mild solution of the system (1.3) in $E_{q, T}$ is a function $\theta \in E_{q, T}$ satisfying

$$
\theta(t)=G(t) \theta_{0}-B(\theta, \theta)(t) \equiv G(t) \theta_{0}-\int_{0}^{t} \nabla G(t-s)(\theta R[\theta])(s) d s
$$

for all $0<t<T$ and $\theta(t) \rightarrow \theta_{0}$ when $t \rightarrow 0^{+}$in $L^{2}\left(\mathbb{R}^{2}\right)$.

Let us remark that the free divergence of the velocity field has been used in the integral form of the solution. Finally, let us point out that this section can be generalized completely for the general 2DQG equation (1.1) in the corresponding homogeneity space $L^{\frac{2}{2 \gamma-1}}\left(\mathbb{R}^{2}\right)$ with $\frac{1}{2}<\gamma \leq 1$.

2.1. Local in time well-posedness. Well-posedness theorems will be a consequence of the following lemma for general Banach spaces:

Lemma 2.4 ([24]). Let $X$ be a Banach space with norm $\|\cdot\|_{X}$, and $B: X \times X \rightarrow X$ be a continuous bilinear map, i.e., there exists $K>0$ such that for all $x_{1}, x_{2} \in X$ we have

$$
\left\|B\left(x_{1}, x_{2}\right)\right\|_{X} \leq K\left\|x_{1}\right\|_{X}\left\|x_{2}\right\|_{X} .
$$

Given $0<\varepsilon<\frac{1}{4 K}$ and $y \in X, y \neq 0$, such that $\|y\|_{X}<\varepsilon$, there exists a solution $x \in X$ for the equation $x=y+B(x, x)$ such that $\|x\|_{X} \leq 2 \varepsilon$. The solution $x$ is unique in the ball $\bar{B}(0,2 \varepsilon)$. Moreover, the solution depends continuously on $y$ in the following sense: If $\|\tilde{y}\|_{X} \leq \varepsilon, \tilde{x}=\tilde{y}+B(\tilde{x}, \tilde{x})$, and $\|\tilde{x}\|_{X} \leq 2 \varepsilon$, then

$$
\|x-\tilde{x}\|_{X} \leq \frac{1}{1-4 K \varepsilon}\|y-\tilde{y}\|_{X} .
$$

We prove the following well-posedness results for mild solutions.

Theorem 2.5. Let $\theta_{0} \in L^{2}$ and $2<q \leq \infty$. There exists $T>0$ such that the initial value problem for (1.3) has a unique mild solution $\theta(t, x) \in E_{q, T}$. Furthermore, assume $\theta_{0} \in L^{1} \cap L^{2}$, then $\theta \in B C\left([0, T), L^{1}\right)$.

By Lemma 2.1, we have already proved that the linear part of the equation can be estimated from the initial data. As a consequence of Lemma 2.4, one needs to verify the continuity in $E_{q, T}$ of the bilinear terms in the integral form of the QG equation to obtain the well-posedness theorems for the solutions of the integral equation. The continuity at $0^{+}$will finally end the proof of well-posedness of mild solutions. For this, we have the following lemma:

Lemma 2.6. Let $2<q \leq \infty, \theta$ and $\phi \in E_{q, T}$. Then

$$
\begin{gathered}
\sup _{0<t<T}\|B(\theta, \phi)\|_{2} \leq K \sup _{0<t<T}\|\theta\|_{2} \sup _{0<t<T} t^{\frac{\alpha}{2}}\|\phi\|_{q}, \\
\sup _{0<t<T} t^{\frac{\alpha}{2}}\|B(\theta, \phi)\|_{q} \leq K \sup _{0<t<T} t^{\frac{\alpha}{2}}\|\theta\|_{q} \sup _{0<t<T} t^{\frac{\alpha}{2}}\|\phi\|_{q} .
\end{gathered}
$$

Moreover, if $\theta$ and $\phi \in B C\left([0, T), L^{1}\left(\mathbb{R}^{2}\right)\right) \cap E_{q, T}$, then

$$
\sup _{0<t<T}\|B(\theta, \phi)\|_{1} \leq C T^{1 / 2} \sup _{0<t<T}\|\theta\|_{2} \sup _{0<t<T}\|\phi\|_{2} .
$$


Proof. Let $q^{\prime}<r \leq q$ and $2<q<\infty$. By estimate (2.1), we have that

$$
\begin{aligned}
\|B(\theta, \phi)\|_{r} & \leq \int_{0}^{t}\|\nabla G(t-s)(R[\theta] \cdot \phi)(s)\|_{r} d s \\
& \leq C \int_{0}^{t}(t-s)^{-\frac{3}{2}+1-\frac{1}{q}}\|(R[\theta] \cdot \phi)(s)\|_{\frac{r+q}{r q}} d s \\
& \leq C \int_{0}^{t}(t-s)^{-\frac{3}{2}+1-\frac{1}{q}}\|\theta(s)\|_{q}\|\phi(s)\|_{r} d s
\end{aligned}
$$

where in the last line we used the continuity of the Riesz transform in $L^{p}$ when $1<p<\infty$. Therefore, doing $r=2$, we obtain

$$
\|B(\theta, \phi)\|_{2} \leq C I(t) \sup _{0<t<T}\|\theta(t)\|_{2} \sup _{0<t<T} t^{\frac{\alpha}{2}}\|\phi(t)\|_{q},
$$

where the integral $I(t)$ in the right-hand side can be computed as

$$
I(t)=\int_{0}^{t}(t-s)^{-\frac{3}{2}+1-\frac{1}{q}} s^{-\frac{\alpha}{2}} d s=\int_{0}^{1}(1-s)^{\frac{\alpha}{2}-1} s^{-\frac{\alpha}{2}} d s=C<\infty .
$$

Now taking $r=q$, we get

$$
\|B(\theta, \phi)\|_{q} \leq C t^{-\frac{\alpha}{2}} \sup _{0<t<T} s^{\frac{\alpha}{2}}\|\theta(s)\|_{q} \sup _{0<t<T} s^{\frac{\alpha}{2}}\|\phi(s)\|_{q},
$$

and thus, we finish the proof of continuity of the bilinear form in $E_{q, T}$. The second part of the lemma follows by

$$
\begin{aligned}
\|B(\theta, \phi)(t)\|_{1} & \leq \int_{0}^{t}\|\nabla g(t-s)\|_{1}\|(R[\theta] \cdot \phi)(s)\|_{1} d s \\
& \leq C \int_{0}^{t}(t-s)^{-\frac{1}{2}}\|\theta(s)\|_{2}\|\phi(s)\|_{2} d s \\
& \leq C T^{\frac{1}{2}} \sup _{0<t<T}\|\theta(s)\|_{2} \sup _{0<t<T}\|\phi(s)\|_{2} .
\end{aligned}
$$

Proof of Theorem 2.5. We will perform the proof in two steps:

Step 1. We start by proving that for $\theta_{0} \in L^{2}\left(\mathbb{R}^{2}\right)$, there exist $T_{1}>0$ and $\varepsilon_{1}>0$ sufficiently small, and a unique solution of integral equation (2.5) such that

$$
\lim _{t \rightarrow 0} t^{\frac{\alpha}{2}}\|\theta\|_{q}=0 \text { and } \sup _{0<t<T_{1}} t^{\frac{\alpha}{2}}\|\theta\|_{q}<2 \varepsilon_{1} .
$$

For this, let us consider the normed space $H_{\varepsilon_{1}}=\left\{\theta(t, x)\right.$ measurable; $\sup _{0<t<T_{1}} t^{\frac{\alpha}{2}}\|\theta\|_{q}<$ $\left.2 \varepsilon_{1}\right\}$ with norm $\sup _{0<t<T_{1}} t^{\frac{\alpha}{2}}\|\cdot\|_{q}$, where $\varepsilon_{1}>0$ and $T_{1}>0$ will be chosen below. Since $\theta_{0} \in L^{2}\left(\mathbb{R}^{2}\right)$ and by using $(2.2)$, for each $\varepsilon_{1}>0$, we can find $T_{1}>0$ sufficiently small, such that

$$
\sup _{0<t<T_{1}} t^{\frac{\alpha}{2}}\left\|G(t) \theta_{0}\right\|_{q}<\varepsilon_{1}
$$

Now, choosing $\varepsilon_{1}>0$ and $T_{1}>0$ small enough, note that by inequality (2.6), we can apply Lemma 2.4 in the space $H_{\varepsilon_{1}, T_{1}}$ to obtain a unique solution $\theta(t, x)$ of integral equation (2.5) in $H_{\varepsilon_{1}, T_{1}}$ with the desired property.

Step 2. To finish the proof of the well-posedness of mild solutions in $E_{q, T}$, it remains to check that $\theta \in B C\left([0, T) ; L^{2}\right)$ with $0<T \leq T_{1}$. 
Let us choose $0<\varepsilon \leq \varepsilon_{1}$ and $0<T \leq T_{1}$ such that $2 K \varepsilon<1$ and $\sup _{0<t<T} t^{\frac{\alpha}{2}}\left\|\theta_{0}\right\|_{q} \leq 2 \varepsilon$. The well-posedness in $H_{\varepsilon}$ shows that $\sup _{0<t<T} t^{\frac{\alpha}{2}}\|\theta\|_{q} \leq 2 \varepsilon$.

Since the solution given by Lemma 2.4 in $H_{\varepsilon}$ is obtained by recursion

$$
\begin{aligned}
\theta_{1}(t, x) & =G(t) \theta_{0}(x), \\
\theta_{k+1}(t, x) & =\theta_{1}(t, x)-B\left(\theta_{k}, \theta_{k}\right),
\end{aligned}
$$

where $k \in N$, we can use Lemmas 2.1 and 2.6 to get

$$
\sup _{0<t<T}\left\|\theta_{1}(t)\right\|_{2} \leq \widetilde{C}\left\|\theta_{0}\right\|_{2}
$$

and

$$
\sup _{0<t<T}\left\|\theta_{k+1}(t)\right\|_{2} \leq \widetilde{C}\left\|\theta_{0}\right\|_{2}+K \sup _{0<t<T}\left\|\theta_{k}(t)\right\|_{2} \sup _{0<t<T} t^{\frac{\alpha}{2}}\left\|\theta_{k}(t)\right\|_{q} .
$$

Therefore, we can estimate

$$
\sup _{0<t<T}\left\|\theta_{k+1}(t)\right\|_{2} \leq \widetilde{C}\left\|\theta_{0}\right\|_{2}+2 K \varepsilon \sup _{0<t<T}\left\|\theta_{k}(t)\right\|_{2}
$$

Let us denote $M_{0}=\widetilde{C}\left\|\theta_{0}(t)\right\|_{2}$ and $M_{k}=\sup _{t>0}\left\|\theta_{k}(t)\right\|_{2}$, then the sequence $\left\{M_{k}\right\}$ satisfies

$$
M_{k+1} \leq M_{0}+2 K \varepsilon M_{k}
$$

Taking $R=2 K \varepsilon<1$, we can write

$$
M_{k} \leq\left(1+R+R^{2}+\cdots+R^{k}\right) M_{0} \leq \frac{1}{1-R} M_{0}
$$

and thus,

$$
w_{k+1}=\theta_{k+1}-\theta_{k}=-B\left(\theta_{k}, \theta_{k}\right)+B\left(\theta_{k-1}, \theta_{k-1}\right)=-B\left(w_{k}, \theta_{k}\right)-B\left(\theta_{k}, w_{k}\right) .
$$

Finally, Lemma 2.6 implies

$$
\sup _{0<t<T}\left\|w_{k+1}\right\|_{2} \leq 2 K \sup _{0<t<T}\left\|\theta_{k}(t)\right\|_{2} \sup _{0<t<T} t^{\frac{\alpha}{2}}\left\|w_{k}(t)\right\|_{q} \leq \frac{2 K}{1-R} M_{0} \sup _{0<t<T} t^{\frac{\alpha}{2}}\left\|w_{k}(t)\right\|_{q}
$$

Since $\lim _{k \rightarrow \infty} t^{\frac{\alpha}{2}}\left\|w_{k}\right\|_{q}=\lim _{n \rightarrow \infty} t^{\frac{\alpha}{2}}\left\|\theta_{k+1}-\theta_{k}\right\|_{q}=0$, then the sequence $\left\{\theta_{k}\right\}$ is a Cauchy sequence in $B C\left([0, \infty) ; L^{2}\right)$ and thus, it converges to $\theta \in B C\left([0, \infty) ; L^{2}\right)$, by uniqueness of the limit in distributional sense.

Adapting the arguments of T. Kato in [23] we can prove that the constructed solutions are $C^{\infty}$-smooth instantaneously. These arguments are included in [4, Proposition 3.15].

Proposition 2.7. Let $\theta(t, x) \in E_{q, T}$ be the unique global mild solution of Theorem 2.5. Then,

$$
\partial_{t}^{k} \partial_{x}^{m} \theta(t, x) \in C\left((0, T), L^{2} \cap L^{q}\right) .
$$

As a consequence, the solution $\theta(t, x)$ is infinitely smooth in space and time.

2.2. Global existence. In order to show that solutions obtained in the previous subsection exist for all $T>0$, we will need global $L^{p}$-bounds on the solutions.

Proposition 2.8. Let $\theta_{0} \in L^{2}$ and $\theta(t, x)$ be the unique global mild solution of Theorem 2.5 with initial data $\theta_{0}$. Then for all $0<t<T$, we have that $\|\theta(t)\|_{2} \leq\left\|\theta_{0}\right\|_{2}$. Moreover, if $\theta_{0} \in L^{1} \cap L^{2}$ then $\|\theta(t)\|_{1} \leq\left\|\theta_{0}\right\|_{1}$ for all $0<t<T$. 
Proof. Let $2 \leq q<\infty$ and let us fix the notation $\theta(t)^{q}=|\theta(t)|^{q-1} \theta(t)$. By Proposition 2.7, the solution $\theta$ is smooth for time $0<t_{1} \leq t<T$ and $\theta(t, x) \rightarrow 0$ when $|x| \rightarrow \infty$. Since that $\nabla \cdot R \theta=0$, we integrate by parts and obtain for $0<t_{1} \leq t<T$

$$
\begin{aligned}
\frac{\partial}{\partial t}\|\theta(t)\|_{q}^{q} & =\int_{\mathbb{R}^{2}} \frac{\partial}{\partial t}|\theta(t)|^{q} d x=q \int_{\mathbb{R}^{2}} \theta(t)^{q-1} \frac{\partial}{\partial t} \theta(t) d x \\
& =q \int_{\mathbb{R}^{2}} \theta(t)^{q-1}\left(\Delta_{x} \theta-R \theta \cdot \nabla_{x} \theta\right) d x \\
& =-q(q-1) \int_{\mathbb{R}^{2}} \theta(t)^{q-2}\left|\nabla_{x} \theta(t)\right|^{2} d x \\
& =-4 \frac{q-1}{q} \int_{\mathbb{R}^{2}}\left|\nabla_{x}\left(\theta^{\frac{q}{2}}\right)\right|^{2} d x .
\end{aligned}
$$

We can obtain an analogous estimate in case we use as test function a regularized version of the sign function; we omit the details since the procedure is fairly standard. Passing to the limit in the regularization parameter we deal with the $q=1$ case. Summarizing,

$$
\|\theta(t)\|_{2} \leq\left\|\theta\left(t_{1}\right)\right\|_{2} \text { and }\|\theta(t)\|_{1} \leq\left\|\theta\left(t_{1}\right)\right\|_{1} .
$$

Now, taking the limit $t_{1} \rightarrow 0$ we conclude the proof.

With the previous maximum principle like estimate, we can extend now our solutions.

Theorem 2.9. Let $\theta$ be the solution of Theorem 2.5 with initial data $\theta_{0} \in L^{2}$. Then, $\theta$ is a global solution of (2.5) and

$$
\theta \in B C\left([0, \infty) ; L^{2}\right) .
$$

Moreover, If $\theta_{0} \in L^{1} \cap L^{2}$, then $\theta$ satisfies

$$
\theta \in B C\left([0, \infty) ; L^{1} \cap L^{2}\right) .
$$

Proof. Let $2<q<\infty$. Note that if $\theta_{0} \in L^{q}$, then

$$
t^{\frac{\alpha}{2}}\left\|G(t) \theta_{0}\right\|_{q} \leq t^{\frac{\alpha}{2}}\left\|\theta_{0}\right\|_{q} \rightarrow 0 \text { when } t \rightarrow 0^{+} .
$$

Therefore, if $\theta_{0} \in L^{2} \cap L^{q}$ the existence time $T>0$ obtained in Theorem 2.5 depends just on the norm $\left\|\theta_{0}\right\|_{q}$. Now, let $\theta_{0} \in L^{2}\left(\mathbb{R}^{2}\right)$. We continue the solution $\theta$, by solving the initial value problem with the initial data $\theta\left(\frac{T}{2}\right)$ and we obtain the extension for $t \in\left[0, \frac{T}{2}+T_{1}\right)$. Now, we start of the time $t=\frac{T+T_{1}}{2}$ and obtain a solution for $t \in\left[0, \frac{T}{2}+\frac{T_{1}}{2}+T_{2}\right)$ and so on. By Theorem 2.5, we know that $\theta\left(\frac{T}{2}\right) \in L^{2} \cap L^{q}$ and by Proposition 2.8, we have that

$$
\|\theta(t)\|_{q} \leq\left\|\theta\left(\frac{T}{2}\right)\right\|_{q} \text { for all } t \geq \frac{T}{2} .
$$

Thus, we have that $T_{1}, T_{2}, \cdots \geq \tilde{T}>0$ where $\tilde{T}>0$ depends only on $\left\|\theta\left(\frac{T}{2}\right)\right\|_{q}$. This concludes the proof.

\subsection{Decay estimates in $L^{p}$}

Proposition 2.10. Let $\theta$ be the solution of Theorem 2.5 with initial data $\theta_{0} \in L^{2}$. If $2 \leq q \leq \infty$, then

$$
t^{\frac{\alpha}{2}} \theta \in B C\left([0, \infty), L^{q}\right)
$$


Moreover, if $1 \leq q \leq \infty$ and $\theta_{0} \in L^{1} \cap L^{2}$, then $\theta$ satisfies

$$
\left.t^{\frac{\alpha}{2}+\frac{1}{2}} \theta \in B C\left([0, \infty) ; L^{q}\right)\right) .
$$

Proof. In order to show the first part, we proceed as in Kato ([21]). By GagliardoNirenberg inequality we have

$$
\|\phi\|_{2} \leq C\|\phi\|_{1}^{\frac{1}{2}}\|\nabla \phi\|_{2}^{\frac{1}{2}}
$$

and if we take $\phi=\theta^{\frac{q}{2}}$ then

$$
\|\theta\|_{q}^{q} \leq C\|\theta\|_{\frac{q}{2}}^{\frac{q}{2}}\left\|\nabla \theta^{\frac{q}{2}}\right\|_{2}
$$

Now, by substituting in (2.9) and using the notation $h_{q}(t)=\|\theta(t)\|_{q}^{q}$, we obtain

$$
\frac{\partial}{\partial t} h_{q} \leq-4 C \frac{q-1}{q}\left(h_{\frac{q}{2}}\right)^{-2} h_{q}^{2}
$$

Since $\|\theta(t)\|_{2} \leq\left\|\theta_{0}\right\|_{2}$, if we take $q=2^{n}$ with $n=2,3, \ldots$, we can solve the differential inequality (2.14) and obtain for all $t>0$

$$
h_{q}(t) \leq M_{q} t^{1-\frac{q}{2}}=M_{q} t^{-\frac{\alpha}{2} q}, \text { where } M_{q}=\frac{q / 2-1}{4 C(1-q)} M_{\frac{q}{2}}^{2} \text { and } M_{2}=\left\|\theta_{0}\right\|_{2} .
$$

Using the fact that $\prod_{q=2}^{\infty}\left(M_{q}\right)^{\frac{1}{q}}<\infty$, it is easy to see that

$$
\|\theta(t)\|_{2^{n}} \leq C\left\|\theta_{0}\right\|_{2}^{\frac{1}{2}} t^{-\frac{1}{2}+\frac{1}{2^{n}}} .
$$

Letting $n \rightarrow \infty$, we get

$$
\|\theta(t)\|_{\infty} \leq C\left\|\theta_{0}\right\|_{2}^{\frac{1}{2}} t^{-\frac{1}{2}}
$$

and by interpolation we obtain for all $2 \leq q \leq \infty$,

$$
\|\theta(t)\|_{q} \leq C\left\|\theta_{0}\right\|_{2}^{\frac{1}{2}} t^{-\frac{\alpha}{2}} .
$$

Now, assume that $\theta_{0} \in L^{1} \cap L^{2}$. Using that $\|\theta(t)\|_{1} \leq\left\|\theta_{0}\right\|_{1}$ and starting the sequence $q=2^{n}$ at $n=1$ (instead of starting at $n=2$ ), we proceed analogously to obtain

$$
\|\theta(t)\|_{q} \leq C\left\|\theta_{0}\right\|_{1} t^{-1+\frac{1}{q}} \leq C\left\|\theta_{0}\right\|_{1} t^{-\frac{\alpha}{2}-\frac{1}{2}} \text { for all } 1<q \leq \infty
$$

finishing the proof.

REMARK 2.11. An important observation is that the velocity field $R[\theta]$ satisfies the same estimate also in case $q=\infty$. In fact, we use the Gagliardo-Nirenberg inequality together with the continuity of the Riesz transform in $L^{q}$ with $1<q<\infty$, to estimate (2.12) with $q=4$, and get

$$
\begin{aligned}
\|R[\theta]\|_{\infty} & \leq C\|R[\theta(t)]\|_{4}^{1 / 2}\|\nabla R[\theta(t)]\|_{4}^{1 / 2} \\
& \leq C\|\theta\|_{4}^{1 / 2}\|\nabla \theta\|_{4}^{1 / 2} \leq C t^{\frac{1}{2}\left(-\frac{1}{4}-\frac{1}{4}-\frac{1}{2}\right)} \\
& =C t^{-\frac{1}{2}}
\end{aligned}
$$

In case $\theta_{0} \in L^{1} \cap L^{2}$, we also obtain

$$
\begin{aligned}
\|R[\theta]\|_{\infty} & \leq C\|\theta\|_{4}^{1 / 2}\|\nabla \theta\|_{4}^{1 / 2} \leq C t^{\frac{1}{2}\left(-\frac{3}{4}-\frac{3}{4}-\frac{1}{2}\right)} \\
& =C\left\|\theta_{0}\right\|_{2}^{1 / 2} t^{-1}
\end{aligned}
$$


Proposition 2.12. Let $\theta$ be the solution of Theorem 2.5 with initial data $\theta_{0} \in L^{2}$. If $k \in \mathbb{N}$ and $2 \leq q \leq \infty$, then

$$
t^{\frac{\alpha}{2}+\frac{k}{2}} \nabla_{x}^{k} \theta \in B C\left([0, \infty) ; L^{q}\right)
$$

holds. Moreover, if $\theta_{0} \in L^{1} \cap L^{2}$ and $1 \leq q \leq \infty$ then $\theta$ satisfies

$$
t^{\frac{\alpha}{2}+\frac{k+1}{2}} \nabla_{x}^{k} \theta \in B C\left([0, \infty) ; L^{q}\right) .
$$

Proof. We proceed by induction. The estimate in case $k=0$ corresponds to (2.15). Now, assume that the inequality (2.17) holds for any integer number $1 \leq n \leq k$. We will prove that is true for $n=k+2 h$, with $0<h \leq \frac{1}{2}$. Let us consider the operator $\partial^{h} \equiv(-\Delta)^{\frac{h}{2}}$. First note that

$$
\left\|\partial^{h}(f g)\right\|_{\frac{q}{2}} \leq C\left(\left\|\partial^{h} f\right\|_{q}\|g\|_{q}+\|f\|_{q}\left\|\partial^{h} g\right\|_{q}\right)
$$

for all $h>0$. Applying this operator in the integral equation (2.5) and calculating the norm $\|\cdot\|_{q}$ (with $q>4$ ) and using (2.15), we obtain

$$
\begin{aligned}
\left\|\partial^{h+k} \theta(t)\right\|_{q} \leq & C t^{-\left(\frac{\alpha}{2}+\frac{h+k}{2}\right)}\left\|\theta_{0}\right\|_{2}+\left\|\int_{t / 2}^{t} \partial^{h}\left(\nabla G(t-s)\left(\partial^{k}(\theta R[\theta])\right)(s)\right) d s\right\|_{q} \\
& +\left\|\int_{0}^{t / 2}\left(\partial^{h+k} \nabla G(t-s)\right)((\theta R[\theta])(s)) d s\right\|_{q} \\
\leq & C t^{-\left(\frac{\alpha}{2}+\frac{h+k}{2}\right)}\left\|\theta_{0}\right\|_{2}+C t^{-\alpha-\frac{k}{2}} \int_{t / 2}^{t}(t-s)^{-\frac{h}{2}+\frac{\alpha}{2}-1} d s \\
& +C t^{-\frac{h+k}{2}+\frac{\alpha}{2}-1} \int_{0}^{t / 2} s^{-\alpha} d s \\
= & C t^{-\left(\frac{\alpha}{2}+\frac{h+k}{2}\right)},
\end{aligned}
$$

where in the second estimate we have split the integral into two pieces and in the second part we have put all the derivatives on the heat kernel.

Proceeding similarly by applying the operator $\partial^{2 h}$ and using (2.19) (with $q>4$ ), we get

$$
\begin{aligned}
\left\|\partial^{k+2 h} \theta(t)\right\|_{q} \leq & C t^{-\left(\frac{\alpha}{2}+\frac{k+2 h}{2}\right)}\left\|\theta_{0}\right\|_{2}+\left\|\int_{0}^{t} \partial^{k+2 h}(\nabla G(t-s)(\theta R[\theta])(s)) d s\right\|_{q} \\
\leq & C t^{-\left(\frac{\alpha}{2}+\frac{k+2 h}{2}\right)}\left\|\theta_{0}\right\|_{2} \\
& +C t^{-\alpha-\frac{k+h}{2}} \int_{t / 2}^{t}(t-s)^{-\frac{h}{2}+\frac{\alpha}{2}-1} d s+C t^{-\frac{k+2 h}{2}+\frac{\alpha}{2}-1} \int_{0}^{t / 2} s^{-\alpha} d s \\
= & C t^{-\left(\frac{\alpha}{2}+\frac{k+2 h}{2}\right)} .
\end{aligned}
$$

We conclude by taking $h=\frac{1}{2}$ and noting that $\left\|\nabla^{k+1} \theta\right\|_{q} \leq C\left\|\partial^{1+k} \theta\right\|_{q}$. The case $q=2$ follows analogously by estimating the norm $\|\nabla \theta\|_{2}$ by the norms $\sup _{t>0}\|\theta\|_{2}$ and $\sup _{t>0} t^{\frac{\alpha}{2}+\frac{1}{2}}\|\nabla \theta\|_{q}($ with $q>4)$.

Now, assume that $\theta_{0} \in L^{1} \cap L^{2}$. We already know that $\|\theta(t)\|_{1} \leq\left\|\theta_{0}\right\|_{1}$ for all $t>0$. Using (2.15) and (2.16) with $\frac{8}{3}<q<4$ instead of only (2.15), applying the operator $\partial$ 
four times with $0<h \leq \frac{1}{4}$ and proceeding analogously, we also get

$$
\left\|\nabla^{k} \theta(t)\right\|_{q} \leq C t^{-\frac{\alpha}{2}-\frac{k}{2}-\frac{1}{2}}
$$

finishing the proof. The general case $(1 \leq q \leq \infty)$ follows by interpolation and similar splitting of the bilinear integral term.

\subsection{Preservation of positivity}

Lemma 2.13. Let $\theta_{0} \in L^{2}$. Assuming $\theta_{0}(x) \geq 0$ not identically zero a.e., then the solution obtained in Theorem 2.5 is positive for all $(t, x) \in(0, \infty) \times \mathbb{R}^{2}$.

Proof. First, assuming that $0 \leq \theta_{0} \in \mathcal{S}\left(\mathbb{R}^{2}\right)$ (Schwartz space), we can prove that the solution $\theta \in B C\left([0, T) ; L^{\infty}\left(\mathbb{R}^{2}\right)\right)$. We also know, by Proposition 2.7 , that the solution $\theta$ satisfies (for all $t_{0}>0$ )

$$
\theta, R \theta \in B C^{2}\left(\left[t_{0}, T\right) \times \mathbb{R}^{2}\right)
$$

and

$$
\theta_{t}-\Delta \theta+R \theta \cdot \nabla \theta=0
$$

By the maximum principle for parabolic equations, we have

$$
\inf _{x \in \mathbb{R}^{2}} \theta\left(t_{0}, x\right) \leq \inf _{x \in \mathbb{R}^{2}} \theta(t, x) \text { for all } t_{0} \leq t<T .
$$

Since $\left\|\theta\left(t_{0}, x\right)-\theta_{0}(x)\right\|_{\infty} \rightarrow 0$ when $t_{0} \rightarrow 0, \lim _{t_{0} \rightarrow 0}\left(\inf _{x \in \mathbb{R}^{2}} \theta\left(t_{0}, x\right)\right)=\inf _{x \in \mathbb{R}^{2}} \theta_{0}(x)$. Therefore letting $t_{0} \rightarrow 0$ in (2.20) we get that $0 \leq \theta(t, x)$ for all $0<t<T$. In the general case $\theta_{0} \in L^{2}$, noting that the convergence in the norm $B C\left([0, T) ; L^{2}\left(\mathbb{R}^{2}\right)\right)$ imply weak convergence that preserves the positivity, by density we also get that $0 \leq \theta(t, x)$ for all $0<t<T$. Now, by the maximum principle, note that in $(2.20), \inf _{x \in \mathbb{R}^{2}} \theta\left(t_{0}, x\right)=$ $\inf _{x \in \mathbb{R}^{2}} \theta(t, x)$ only when $\theta\left(t_{0}, x\right)=\theta(t, x)$. And since $(2.20)$ holds for all $t_{0}>0$, we get that $\theta(t, x)>0$, unless $\theta_{0}(x)=0$ almost everywhere.

\section{Global existence of smooth fast-decaying solutions in self-similar variables.} We introduce the self-similar variables:

$$
\left\{\begin{array}{c}
y=x(1+2 t)^{-\frac{1}{2}} \\
\tau=\frac{1}{2} \log (1+2 t)
\end{array} \text { for all } t>0 \text { and } x \in \mathbb{R}^{2} .\right.
$$

If $\theta(t, x)$ is a solution of system (1.3) then the function

$$
\psi(\tau, y)=(2 t+1) \theta(x, t)
$$

satisfies

$$
\frac{\partial}{\partial \tau} \psi(\tau, y)=\mathcal{L} \psi-e^{-\tau}\left(R \psi \cdot \nabla_{y} \psi\right)
$$

where

$$
\mathcal{L} \psi=\nabla_{y} \cdot\left(\nabla_{y} \psi+y \psi\right)
$$

denotes the well-known Fokker-Planck operator. Thus, we can write the system (1.3) in the new variables as

$$
\begin{aligned}
\frac{\partial}{\partial \tau} \psi(\tau, y) & =\mathcal{L} \psi-e^{-\tau}\left(R \psi \cdot \nabla_{y} \psi\right) & & \text { for all } \tau>0 \text { and } y \in \mathbb{R}^{2}, \\
\psi(0, y) & =\theta_{0} & & \text { for all } y \in \mathbb{R}^{2} .
\end{aligned}
$$


Let us note that the change of variable transform the Oseen-vortex solutions corresponding to the time translation of the heat kernel $G(x, 2 t+1)$ into the gaussian function, which is a stationary solution of the linear Fokker-Planck equation $\partial_{\tau} \psi-\mathcal{L} \psi=0$. These self-similar changes of variables have been widely used to study large time asymptotics of nonlinear diffusion equations [6] and 2D Navier-Stokes equations [15, 16].

We denote by $S(\tau)=e^{\tau \mathcal{L}}$ the semigroup generated by the operator $\mathcal{L} \psi=\nabla_{y} \cdot\left(\nabla_{y} \psi+\right.$ $y \psi)$. Since $\nabla(\mathcal{L} \psi)=(\mathcal{L}+1) \nabla \psi$ we have

$$
\nabla S(\tau) f=e^{-\tau} e^{\tau \mathcal{L}} \nabla f
$$

Let us define the weighted Lebesgue space as $L^{r}(m)=\left\{h \in L^{r}\left(\mathbb{R}^{2}\right) ;\|h\|_{m, r}<\infty\right\}$ with $\|h\|_{r, m}=\left\|b^{m} h\right\|_{r}$ and $b(y)=\left(1+|y|^{2}\right)^{\frac{1}{2}}$.

We can rephrase some of the results in [16] on the Fokker-Planck operator:

1. Let $m>1$. There exists $C>0$ such that, for all $f \in L^{2}(m)$,

$$
\|S(\tau) f\|_{m} \leq C\|f\|_{m}, \quad\|\nabla S(\tau) f\|_{m} \leq C a(\tau)^{-\frac{1}{2}}\|f\|_{m}, \tau>0
$$

where $a(\tau)=\frac{1-e^{-2 \tau}}{2}$.

2. If $0<\mu \leq \frac{1}{2}$ and $m>1+2 \mu$, there exists $C>0$ such that, for all $f \in L^{2}(m)$ with $\int_{\mathbb{R}^{2}} f(y) d y=0$,

$$
\|S(\tau) f\|_{m} \leq C e^{-2 \mu \tau}\|f\|_{m}, \quad\|\nabla S(\tau) f\|_{m} \leq C a(\tau)^{-\frac{1}{2}} e^{-2 \mu \tau}\|f\|_{m}, \tau>0 .
$$

$3.1 \leq l \leq r \leq \infty$ and $m \geq 0$. There exists $C>0$ such that

$$
\left\||y|^{m} \nabla^{k} S(\tau) f\right\|_{r} \leq C e^{2 \tau\left(1-\frac{1}{l}-\frac{m}{2}\right)} a(\tau)^{-\left(\frac{1}{l}-\frac{1}{r}\right)-\frac{|k|}{2}}\left\|b(y)^{m} f\right\|_{l}, 0<\tau<\infty .
$$

We recall that the field velocity of quasi-geostrophic equation is determined from the potential temperature $\theta$ through the operator $u=R[\theta]=\left(-R_{2} \theta, R_{1} \theta\right)$ where $R_{i}$ are the Riesz transforms. The next lemma deals with the continuity of Riesz transform in $L^{r}(m)$ spaces and it is a direct application of [17, Theorem 3.1, Chapter IV, p. 411].

LEMma 3.1. The Riesz transform $R_{j}=\partial_{j}(-\Delta)^{-\frac{1}{2}}, j=1,2, \ldots, n$, is continuous in $L^{r}(m), 1<r<\infty$ and $0 \leq m<\infty$.

Before starting the analysis of the system (3.2)-(3.3) in $L^{2}(m)$, let us remark that we have already obtained global existence of solutions for the system in $L^{2}\left(\mathbb{R}^{2}\right)$. Indeed, this follows from Theorem 2.9 together with the change of variables (3.1). Moreover, by Proposition 2.10 and Proposition 2.12, we have the following estimate:

$$
a(\tau)^{\frac{\alpha}{2}+\frac{1}{2}+\frac{k}{2}} \nabla^{k} \psi(\tau) \in B C\left((0, \infty) ; L^{q}\left(\mathbb{R}^{2}\right)\right) \text { for all } 1 \leq q \leq \infty, \text { for } \theta_{0} \in L^{1} \cap L^{2},
$$

where $\alpha=1-\frac{2}{q}$ and $k \in \mathbb{N}$.

Although we could directly work with the solution already constructed, we prefer to give a meaning to solutions in the weighted spaces and then, identify them with the previous solution by uniqueness in $L^{2}\left(\mathbb{R}^{2}\right)$. Now, let us introduce suitable function spaces to analyze the Cauchy problem for system (3.2)-(3.3) based on weighted spaces $L^{r}(m)$. Let us consider $H_{q, m, T}$ the space of all functions $h(\tau, y), \tau>0$ and $x \in \mathbb{R}^{2}$, such that

$$
\begin{aligned}
h(\tau, y) & \in B C\left([0, T), L^{2}(m)\right), \\
a(\tau)^{\frac{\alpha}{2}} h(\tau, y) & \in B C\left([0, T), L^{q}(m)\right),
\end{aligned}
$$


where $\alpha=1-\frac{2}{q}$. The norm in $H_{q, m, T}$ is naturally defined by

$$
\|h\|_{H_{q, m, T}}=\sup _{0<t<T} a(\tau)^{\frac{\alpha}{2}}\|h(\tau, y)\|_{m, q}+\sup _{0<t<T}\|h\|_{m, 2} .
$$

A mild solution of the system (3.2)-(3.3) in $H_{q, m, T}$ is a function $\psi(t)$ in the corresponding space satisfying

$$
\psi(\tau)=S(\tau) \theta_{0}-B_{S}(\theta, \theta)(\tau) \equiv S(\tau) \theta_{0}-\int_{0}^{\tau} e^{-(\tau-s)} \nabla S(\tau-s)\left(e^{-s} \psi R[\psi]\right)(s) d s
$$

for all $0<\tau<T$. Note that we have used the equality (3.4) in this formulation.

TheOREM 3.2. Let $\theta_{0} \in L^{2}(m)$. There exists $T>0$ such that the initial value problem (3.2)-(3.3) has a unique mild solution $\psi(\tau, y) \in H_{q, m, T}$.

The result follows analogous arguments in the previous section, thus, we will only emphasize the main new points. We start by showing the continuity in $H_{q, m, T}$ of the bilinear term of the integral equations (3.9) in the mild formulation.

LEMmA 3.3. Let $\psi$ and $\varphi \in H_{q, m, T}$. Then

$$
\begin{gathered}
\sup _{0<\tau<T}\left\|B_{S}(\psi, \varphi)\right\|_{2, m} \leq C \sup _{0<\tau<T}\|\psi\|_{2, m} \sup _{0<\tau<T} a(\tau)^{\frac{\alpha}{2}}\|\varphi\|_{q, m}, \\
\sup _{0<\tau<T} a(\tau)^{\frac{\alpha}{2}}\left\|B_{S}(\psi, \varphi)\right\|_{q, m} \leq C \sup _{0<\tau<T} a(\tau)^{\frac{\alpha}{2}}\|\psi\|_{q, m} \sup _{0<\tau<T} a(\tau)^{\frac{\alpha}{2}}\|\varphi\|_{q, m} .
\end{gathered}
$$

Moreover, if $\psi$ and $\varphi \in B C\left([0, T), L^{p}(m)\right) \cap H_{q, m, T}$ then

$$
\sup _{0<\tau<T}\left\|B_{S}(\psi, \varphi)\right\|_{p, m} \leq C \sup _{0<\tau<T}\|\psi\|_{p, m} \sup _{0<\tau<T} a(\tau)^{\frac{\alpha}{2}}\|\varphi\|_{q, m} .
$$

Proof. Using $\frac{1}{l}=\frac{1}{r}+\frac{1}{q} \leq 1$ in (3.7), we find

$$
\begin{aligned}
\left\|B_{S}(\psi, \varphi)\right\|_{r, m} & \leq \int_{0}^{\tau} e^{-(\tau-s)} e^{-s}\|\nabla S(\tau-s)(R[\psi] \cdot \varphi)(s)\|_{r, m} d s \\
& \leq \int_{0}^{\tau} e^{-\tau} a(\tau-s)^{\left(\frac{1}{r}-\frac{1}{l}\right)-\frac{1}{2}}\|(R[\psi] \cdot \varphi)(s)\|_{l, m} d s \\
& \leq C \int_{0}^{\tau} e^{-\tau} a(\tau-s)^{\frac{\alpha}{2}-1}\|\psi(s)\|_{q}\|\varphi(s)\|_{r, m} d s,
\end{aligned}
$$

where in the last line we used the continuity of the Riesz transform in $L^{q}\left(\mathbb{R}^{2}\right)$. Setting $r=r$ and $r=q$ in (3.10), and noting that $\|\psi(s)\|_{q} \leq\|\psi(s)\|_{q, m}$, we get

$$
\begin{aligned}
& \left\|B_{S}(\psi, \varphi)\right\|_{r, m} \leq C I_{r}(\tau) \sup _{0<\tau<T}\|\psi(s)\|_{r, m} \sup _{0<\tau<T} a(\tau)^{\frac{\alpha}{2}}\|\varphi(s)\|_{q, m}, \\
& \left\|B_{S}(\psi, \varphi)\right\|_{q, m} \leq C I_{q}(\tau) \sup _{0<\tau<T} a(\tau)^{\frac{\alpha}{2}}\|\psi(s)\|_{q, m} \sup _{0<\tau<T} a(\tau)^{\frac{\alpha}{2}}\|\varphi(s)\|_{q, m},
\end{aligned}
$$

where the integral $I_{r}(\tau)$ and $I_{q}(\tau)$ in the right-hand side can be computed as

$$
\begin{aligned}
I_{r}(\tau)=\int_{0}^{\tau} e^{-\tau} a(\tau-s)^{\frac{\alpha}{2}-1} a(s)^{-\frac{\alpha}{2}} d s & \leq e^{\tau} \int_{0}^{t} \frac{1}{1+2 w}(t-w)^{\frac{\alpha}{2}-1} w^{-\frac{\alpha}{2}} d w \\
& =C e^{-(1-\alpha) \tau}<\infty
\end{aligned}
$$


and

$$
I_{q}(\tau)=\int_{0}^{\tau} e^{-\tau} a(\tau-s)^{\frac{\alpha}{2}-1} a(s)^{-\alpha} d s \leq C a(\tau)^{-\frac{\alpha}{2}} .
$$

Taking $r=2, p$ and $r=q$ in (3.11) finishes the proof of continuity of the bilinear form in $H_{q, m, T}$ and in $B C\left([0, T), L^{p}(m)\right) \cap H_{q, m, T}$.

It remains to check that the linear part of the equation can be estimated from the initial data. In fact, given $\theta_{0} \in L^{2}(m)$ then

$$
\left\|S(\tau) \theta_{0}\right\|_{2, m} \leq C\left\|\theta_{0}\right\|_{2, m} \text { and } \lim _{t \rightarrow 0} a(\tau)^{\frac{\alpha}{2}}\left\|S(\tau) \theta_{0}\right\|_{q, m}=0
$$

We omit the proof since it is analogous to the proof of Lemma 2.1 by using the inequality (3.5) and noting that the set $C_{c}^{\infty}$ is dense in $L^{r}(m)$ for $1 \leq r<\infty$. Theorem 3.2 follows from Lemma 3.3 and the previous property in the same spirit as Theorem 2.5. In order to extend our local solutions to global ones in the weighted space, we need to show the propagation of moments.

Proposition 3.4. Let $m>1, \theta_{0} \in L^{2}(m)$ and $2 \leq r<\infty$. Let $\psi(t, x)$ be the solution of equation (3.2) with initial data $\theta_{0}$. Then, there exist $\tau_{1}>0, \delta>0$ and $C=C\left(\tau_{1},\left\|\theta_{0}\right\|_{1}\right)$, such that, for all $\tau_{1} \leq \tau$, we have

$$
\|\psi(\tau)\|_{r, m}^{r} \leq\left\|\psi\left(\tau_{1}\right)\right\|_{r, m}^{r} e^{-\delta \tau}+C
$$

Proof. Consider the following notation $\psi^{p}=\psi|\psi|^{p-1}$, if $p$ is odd and $\psi^{p}=|\psi|^{p}$ if $p$ is even. Note that $\partial_{x} \psi^{p}=p \psi^{p-1}$. Without loss generality, assume that $2 \leq r<\infty$ be even. Then

$$
\begin{aligned}
\frac{\partial}{\partial \tau} \int_{\mathbb{R}^{2}}|y|^{r m}|\psi(\tau, y)|^{r} d y & =\frac{\partial}{\partial \tau} \int_{\mathbb{R}^{2}}|y|^{r m} r \psi^{r-1} \psi_{\tau} d y \\
& =\int_{\mathbb{R}^{2}}|y|^{r m} r \psi^{r-1}\left[\Delta \psi+y \cdot \nabla_{y} \psi+2 \psi-e^{-\tau}\left(R \psi \cdot \nabla_{y} \psi\right)\right] d y
\end{aligned}
$$

Integration by parts shows that

$$
\begin{aligned}
\int_{\mathbb{R}^{2}}|y|^{r m} r \psi^{r-1} \Delta \psi d y & =-4(1-1 / r) \int_{\mathbb{R}^{2}}|y|^{r m}\left|\nabla \psi^{r / 2}\right|^{2} d y-\int_{\mathbb{R}^{2}} \nabla_{y}\left(|y|^{r m}\right) \cdot \nabla \psi^{r} d y \\
& =-4(1-1 / r) \int_{\mathbb{R}^{2}}|y|^{r m}\left|\nabla \psi^{r / 2}\right|^{2} d y+(r m)^{2} \int_{R^{2}}|y|^{r m-2} \psi^{r} d y \\
\int_{\mathbb{R}^{2}}|y|^{r m} y \cdot \nabla_{y} \psi r \psi^{r-1} d y & =\int_{\mathbb{R}^{2}}|y|^{r m} y \cdot \nabla \psi^{r} d y=-(r m+2) \int_{\mathbb{R}^{2}}|y|^{r m} \psi^{r} d y
\end{aligned}
$$

and

$$
\begin{aligned}
-e^{-\tau} \int_{\mathbb{R}^{2}}|y|^{r m} r \psi^{r-1}(R \psi \nabla \psi) d y & =-e^{-\tau} \int_{\mathbb{R}^{2}}|y|^{r m} \nabla \cdot\left(R \psi \psi^{r}\right) d y \\
& =e^{-\tau} \int_{\mathbb{R}^{2}} r m|y|^{r m-2}(y \cdot R \psi) \psi^{r} d y
\end{aligned}
$$


Note that $L^{2}(m) \subset L^{1} \cap L^{2}$ for $m>1$. Taking $\tau \geq \tau_{1}>0$, by estimate (3.8), we get the following estimates

$$
\begin{aligned}
& \|R \psi\|_{\infty} \leq C a(\tau)^{-1} \leq C a\left(\tau_{1}\right)^{-1}, \\
& \|\psi(\tau)\|_{r} \leq C a(\tau)^{-\left(1-\frac{1}{r}\right)} \leq C .
\end{aligned}
$$

We also recall that, for each $\varepsilon>0$ there exists $C_{\varepsilon}$, such that $|y|^{r m-1},|y|^{r m-2} \leq$ $C_{\varepsilon}+\varepsilon|y|^{r m}$. Thus, taking $\varepsilon>0$ sufficiently small, we get

$$
\frac{\partial}{\partial \tau} \int_{\mathbb{R}^{2}}|y|^{r m}|\psi|^{r} d y \leq-\delta \int_{\mathbb{R}^{2}}|y|^{r m} \psi^{r} d y+C \int_{\mathbb{R}^{2}} \psi^{r} d y,
$$

which implies that

$$
\|\psi(\tau)\|_{r, m}^{r} \leq\left\|\psi\left(\tau_{1}\right)\right\|_{r, m}^{r} e^{-\delta t}+C\left(1+e^{-\delta t}\right)
$$

by Gronwall Lemma.

We can now extend our solutions to infinity in time and we are able to get some regularity properties.

TheOREM 3.5. Let $m>1$ and $2 \leq q<\infty$. Let $\psi$ be the solution of Theorem 3.2 with initial data $\theta_{0} \in L^{2}(m)$. Then $\psi$ can be continued to a global solution of (3.9) and we have

$$
a(\tau)^{\frac{\alpha}{2}} \psi(\tau) \in B C\left([0, \infty) ; L^{q}(m)\right) \text { and } a(\tau) \nabla \psi \in B C\left([0, \infty), L^{2}(m)\right) .
$$

Proof. Since $\theta_{0} \in L^{2}(m) \subset L^{1} \cap L^{2}$, there exists a global solution $\psi(\tau, y)$ of system (3.2)-(3.3) satisfying

$$
a(\tau)^{\frac{\alpha}{2}+\frac{1}{2}} \psi(\tau) \in B C\left((0, \infty) ; L^{q}\left(\mathbb{R}^{2}\right)\right) \text { for all } 1<q \leq \infty
$$

as it was discussed above. Now, using the Proposition 3.4 with $\tau_{1}=T / 2$, we obtain that

$$
\psi \in B C\left((T / 2, \infty) ; L^{q}(m)\right) .
$$

Thus, we finish the proof of first part in (3.13), by observing that $\psi \in H_{q, m T}$ and $a(\tau) \rightarrow 1 / 2$ when $\tau \rightarrow \infty$. that

In order to show the second statement, let $2<q<\infty$. We recall that $\alpha=1-\frac{2}{q}$. Note

$$
\|\phi \varphi\|_{\frac{2 q}{q+2}, m} \leq\|\phi\|_{2, m}\|\varphi\|_{q}
$$

Since $a(\tau) \rightarrow \frac{1}{2}$ when $\tau \rightarrow \infty$, there exists $C>0$ such that $a(\tau)^{-\frac{1}{2}} \leq C a(\tau)^{-1}$ for all $\tau>0$. With these considerations, calculating the gradient $\nabla$ in the integral equation (3.9), and estimating the norm $\|\cdot\|_{2, m}$, we get

$$
\begin{aligned}
\|\nabla \psi(\tau)\|_{2, m} & \leq\left\|\nabla S(\tau) \theta_{0}\right\|_{2, m}+\left\|\int_{0}^{\tau} e^{-\tau} \nabla((\nabla S(\tau-s))(\psi(s) \cdot R[\psi(s)])) d s\right\|_{2, m} \\
& \leq C a(\tau)^{-\frac{1}{2}}+I_{1}+I_{2} .
\end{aligned}
$$


We estimate each term as

$$
\begin{aligned}
I_{1} & =\left\|\int_{\tau / 2}^{\tau} e^{-\tau}(\nabla S(\tau-s))(\nabla \psi(s) \cdot R[\psi(s)]+\psi(s) \nabla \cdot(R[\psi(s)])) d s\right\|_{2, m} \\
& \leq C\left(\int_{\tau / 2}^{\tau} e^{-\tau} a(\tau-s)^{\frac{\alpha}{2}-1} a(s)^{-\frac{\alpha}{2}-1} d s\right) \sup _{s>0} a(s)^{\frac{\alpha}{2}+1}\|\nabla \psi(s)\|_{q} \sup _{s>0}\|\psi(s)\|_{2, m},
\end{aligned}
$$

and

$$
\begin{aligned}
I_{2} & =\left\|\int_{0}^{\tau / 2} e^{-\tau}(\nabla \nabla S(\tau-s))(\psi(s) R[\psi(s)]) d s\right\|_{2, m} \\
& \leq C\left(\int_{0}^{\tau / 2} e^{-\tau} a(\tau-s)^{\frac{\alpha}{2}-1-\frac{1}{2}} a(s)^{-\frac{\alpha}{2}-\frac{1}{2}} d s\right) \sup _{s>0} a(s)^{\frac{\alpha}{2}+\frac{1}{2}}\|\psi(s)\|_{q} \sup _{s>0}\|\psi(s)\|_{2, m},
\end{aligned}
$$

giving finally

$$
\|\nabla \psi(\tau)\|_{2, m} \leq C\left(a(\tau)^{-\frac{1}{2}}+e^{\tau}\left(a(\tau) e^{2 \tau}\right)^{-1}+e^{2 \tau}\left(a(\tau) e^{2 \tau}\right)^{-1}=C a(\tau)^{-1}\right.
$$

which leads to (3.13).

4. Global convergence. Now, we can use the bounds obtained in previous sections to analyze the asymptotic behavior under different assumptions on the initial data. In the first two cases we will show by direct estimates of the bilinear term that asymptotically the system behaves like the linear heat equation or the linear Fokker-Planck equation depending on the variables in which we state the result. This asymptotic simplification will be shown first for initial data in $L^{1} \cap L^{2}$ and later in $L^{2}(m)$ regardless of their sign. Moreover, we obtain in the last subsection optimal rates of decay for signed initial data by entropy methods.

4.1. Global convergence for $L^{1} \cap L^{2}$ data

THEOREM 4.1. Let $\theta$ be the global solution of Theorem 2.5 with initial data $\theta_{0} \in L^{1} \cap L^{2}$. Let $0 \leq \varepsilon<1,1 \leq q \leq \infty$ and $k \in \mathbb{N}$. Then

$$
\left\|\nabla_{x}^{k} B(\theta, \theta)\right\|_{q} \leq C t^{-\left(\frac{k}{2}+\frac{\alpha}{2}+\frac{1}{2}\right)-\frac{\varepsilon}{2}}
$$

As a consequence, there exists $C>0$ such that

$$
\left\|\theta(t, x)-G(t) \theta_{0}\right\|_{L^{1}\left(\mathbb{R}^{2}\right)} \leq C t^{-\varepsilon / 2} .
$$

Proof. First we deal with the case $2 \leq q \leq \infty$. By estimates (2.17) and (2.18), note that for any $0 \leq \varepsilon<1,2 \leq q \leq \infty$ and $k \in \mathbb{N}$, we have

$$
t^{\frac{\alpha}{2}+\frac{\varepsilon}{2}+\frac{k+1}{2}} \nabla_{x}^{k} \theta \in B C\left([0, \infty) ; L^{q}\right) .
$$

As in the proof of Proposition 2.12, we split the bilinear term in two parts and use the estimate (4.1) to obtain

$$
\begin{aligned}
\left\|\nabla^{k} B(\theta, \theta)\right\|_{q} \leq & \left\|\int_{t / 2}^{t}\left(\nabla G(t-s)\left(\partial^{k}(\theta R[\theta])\right)(s)\right) d s\right\|_{q} \\
& +\left\|\int_{0}^{t / 2}\left(\partial^{k} \nabla G(t-s)\right)((\theta R[\theta])(s)) d s\right\|_{q}=I_{1}+I_{2} .
\end{aligned}
$$


Let $\beta=1-\frac{2}{r}$. We estimate $I_{1}$ with $q^{\prime} \leq r<\infty$ as

$$
\begin{aligned}
I_{1} \leq & C t^{-\frac{\alpha}{2}-\frac{\beta}{2}-\frac{k}{2}-\frac{1+\varepsilon}{2}}\left(\int_{t / 2}^{t}(t-s)^{\frac{\beta}{2}-1} d s\right) \\
& \left.\times \sum_{i=0}^{k} \sup _{t>0} t^{\frac{\alpha+k-i}{2}+\frac{\varepsilon}{2}}\left\|\nabla^{k-i} \theta(t)\right\|_{q} \sup _{t>0} t^{\frac{\beta+k+i}{2}+\frac{1}{2}}\left\|\nabla^{i} \theta(t)\right\|_{r}\right) \\
\leq & C t^{-\frac{\alpha}{2}-\frac{k}{2}-\frac{1+\varepsilon}{2}} .
\end{aligned}
$$

For $I_{2}$, take $r \geq 1$ such that $\frac{1+\varepsilon}{2}<\frac{1}{q}+\frac{1}{r}<1$ and note that $\frac{\beta+\alpha}{2}+\frac{1+\varepsilon}{2}<1$.

$$
\begin{aligned}
I_{2} & \leq C t^{\frac{\beta}{2}-\frac{k}{2}-1} \int_{0}^{t / 2} s^{-\frac{\beta+\alpha}{2}-\frac{1+\varepsilon}{2}} d s\left(\sup _{t>0} t^{\frac{\alpha}{2}+\frac{\varepsilon}{2}}\|\theta(t)\|_{q} \sup _{t>0} t^{\frac{\beta}{2}+\frac{1}{2}}\|\theta(t)\|_{r}\right) \\
& \leq C t^{-\left(\frac{k}{2}+\frac{\alpha}{2}+\frac{1+\varepsilon}{2}\right)} .
\end{aligned}
$$

For the case $1 \leq q<2$ it is enough to show it for $q=1$ and $k=0$, and thus, the complete proof follows from interpolation in Lebesgue space together with similar arguments as above. For this, we use (4.1) with $q=2,0 \leq \varepsilon<1$ and $k=0$ concluding the estimate

$$
\begin{aligned}
\|B(\theta, \theta)\|_{1} & \leq \int_{0}^{t}(t-s)^{-\frac{1}{2}}\|((\theta R[\theta])(s))\|_{1} d s \leq \int_{0}^{t}(t-s)^{-\frac{1}{2}}\|\theta\|_{2}\|\theta\|_{2} d s \\
& \leq \int_{0}^{t}(t-s)^{-\frac{1}{2}} s^{-\frac{1+\varepsilon}{2}} d s \sup _{t>0} t^{\frac{1}{2}}\|\theta\|_{2} \sup _{t>0} t^{\frac{\varepsilon}{2}}\|\theta\|_{2} \leq C t^{-\frac{\varepsilon}{2}} .
\end{aligned}
$$

The final statement is a simple consequence for $q=1$ and $k=0$.

4.2. Global convergence for $L^{2}(m)$ data

Theorem 4.2. Let $m>1,2<q \leq \infty$ and $\theta_{0} \in L^{2}(m)$. Let $\psi \in B C\left([0, \infty) ; L^{2}(m)\right)$ be the solution of the initial value problem (3.2)-(3.3), then

$$
\left\|\psi(\tau)-S(\tau) \theta_{0}\right\|_{L^{2}(m)} \leq C e^{-(1-2 \alpha) \tau}
$$

for all $\tau \geq 0$ and $2 \leq q<\infty$. Taking $q$ close enough to 2 , we conclude the asymptotic simplification in $L^{2}(m)$.

Proof. The estimate (4.2) can be obtained from the same arguments done for (3.12) taking into account the global in time estimate of moments (3.13) with $q=2$ and $q>2$ together with the inequality (3.7), to obtain

$$
\left\|b^{m} B_{S}(\psi, \psi)\right\|_{L^{2}} \leq C e^{\tau \alpha} e^{-(1-\alpha) \tau} \leq C e^{-(1-2 \alpha) \tau}
$$

4.3. Global convergence for positive solutions. We will finally show that our system in self-similar variables (3.2)-(3.3) has one important Liapunov functional that coincides with the one obtained in [15] for the two-dimensional Navier-Stokes equations. Let us define

$$
\Sigma_{\eta} \equiv\left\{\psi \in L_{+}^{1}\left(\mathbb{R}^{2}\right) ; \Phi(\psi)=\eta\right\} \text { with } \eta>0
$$


and consider the relative entropy functional $H_{\eta}: L^{2}(m) \cap \Sigma_{\eta} \rightarrow \mathbb{R}$ by

$$
H_{\eta}(\psi)=\int_{\mathbb{R}^{2}} \psi(y) \log \left(\frac{\psi(y)}{\eta g(y)}\right) d y,
$$

where $g(y)=\frac{1}{2 \pi} e^{-\frac{|y|^{2}}{2}}$.

LEMMA 4.3. Let $m>3$ and $\theta_{0} \in L^{2}(m) \cap \Sigma_{\eta}$. Let $\psi \in B C\left([0, \infty) ; L^{2}(m)\right)$ be the solution of the initial value problem (3.2)-(3.3). Then $H_{\eta}(\psi(\tau)) \leq H_{\eta}\left(\theta_{0}\right)$ for all $\tau \geq 0$ and equality holds for all $\tau \geq 0$ if and only if $\theta_{0}=\eta g$.

Proof. All properties that we need to differentiate the functional $H$ along the trajectory $\{\psi(\tau)\}_{\tau>0}$ were already proved. We know that $\psi(\tau)$ satisfies

$$
\frac{\partial}{\partial \tau} \psi(\tau, y)=\mathcal{L} \psi-e^{-\tau}\left(R \psi \cdot \nabla_{y} \psi\right)
$$

We recall that $R[\psi]=\left(-R_{2} \psi, R_{1} \psi\right)$ where

$$
\left(R_{i} \psi\right)(y)=\int_{\mathbb{R}^{2}} \frac{(y-z)_{i}}{|y-z|^{3}} \psi(z) d z, i=1,2 .
$$

For all $y \in \mathbb{R}^{2}$, denote $y^{\perp}=\left(-y_{2}, y_{1}\right)$. Thus,

$$
\begin{aligned}
\int_{\mathbb{R}^{2}} e^{-\tau}(y \cdot R \psi) \psi & =e^{-\tau} \int_{\mathbb{R}^{2}} \int_{\mathbb{R}^{2}}\left(y \cdot \frac{(y-z)^{\perp}}{|y-z|^{3}}\right) \psi(y) d z d y \\
& =\frac{1}{2} e^{-\tau} \int_{\mathbb{R}^{2}} \int_{\mathbb{R}^{2}}\left((y-z) \cdot \frac{(y-z)^{\perp}}{|y-z|^{3}}\right) \psi(y) d z d y=0 .
\end{aligned}
$$

With this, we can just compute the derivative of the relative entropy functional and the contribution of the nonlinear terms disappears as in [15]. Therefore, we prove as for the linear Fokker-Planck equation [30] that for $0<\tau_{0} \leq \tau$,

$$
H_{\eta}(\psi(\tau))-H_{\eta}\left(\psi\left(\tau_{0}\right)\right)=-\int_{\tau_{0}}^{\tau} I_{\eta}(\psi(s)) d s,
$$

where the entropy dissipation is given by

$$
I_{\eta}(\psi)=\int_{\mathbb{R}^{2}} \psi(y)\left|\nabla \log \left(\frac{\psi(y)}{\eta g(y)}\right)\right|^{2} d y .
$$

Using that the Fisher information $I_{\eta}(\zeta)$ vanishes if and only if $\psi=\eta g$, we conclude the proof.

From the previous Lemma, we deduce the following theorem.

TheOREM 4.4. Let $m>3$ and $\theta_{0} \in L^{2}(m) \cap \Sigma_{\eta}$. Let $\psi \in B C\left([0, \infty) ; L^{2}(m)\right)$ be the solution of the initial value problem (3.2)-(3.3), then

$$
\|\psi(\tau)-\eta g\|_{L^{1}\left(\mathbb{R}^{2}\right)} \leq C e^{-\tau}
$$

with $C=(2 \eta)^{1 / 2} H_{\eta}\left(\theta_{0}\right)^{1 / 2}$. As a consequence of the change of variables (3.1), we conclude

$$
\|\theta(t)-\eta G(t)\|_{L^{1}\left(\mathbb{R}^{2}\right)} \leq C t^{-1 / 2}
$$


Proof. Using Lemma 4.3, we have

$$
\frac{d}{d \tau} H_{\eta}(\psi(\tau))=-I_{\eta}(\psi(\tau))
$$

The Csiszár-Kullback inequality and the Stam-Gross logarithmic Sobolev inequality $[5,30]$ give us, respectively,

$$
\frac{1}{2 \eta}\|\psi-\eta g\|_{L^{1}}^{2} \leq H_{\eta}(\psi) \leq \frac{1}{2} I_{\eta}(\psi)
$$

where $I_{\eta}(\psi)$ already has been defined in (4.3). Therefore, we obtain

$$
\frac{d}{d \tau} H_{\eta}(\psi(\tau))=-I_{\eta}(\psi(\tau)) \leq-2 H_{\eta}(\psi(\tau))
$$

and by Gronwall inequality, we get, for $\tau \geq 0$, the estimate

$$
H_{\eta}(\psi(\tau)) \leq H_{\eta}\left(\theta_{0}\right) e^{-2 \tau} \text {. }
$$

Finally, using the first inequality in (4.4), we get

$$
\|\psi-\eta g\|_{L^{1}} \leq(2 \eta)^{1 / 2} H_{\eta}\left(\theta_{0}\right)^{1 / 2} e^{-\tau} .
$$

Now, the final statement comes from the change of variables (3.1).

The previous theorem shows that the entropy method gives a quick elegant proof of the decay rate towards self-similarity with explicit nice constants in the case of signed initial data. Moreover, it is obvious that the decay rate is optimal by comparing two delayed Oseen vortex solutions. Putting together the information of previous subsections, we can write the following result for unsigned initial data.

Corollary 4.5. Let $m>3, \theta_{0} \in L^{2}(m), \eta=\int_{\mathbb{R}^{2}} \theta_{0}(x) d x$ and $0 \leq \varepsilon<1$. Let $\theta \in$ $B C\left([0, \infty) ; L^{1} \cap L^{2}\right)$ be the solution of the initial value problem $(1.3)$, then

$$
\|\theta(t)-\eta G(t)\|_{L^{1}} \leq C t^{-\frac{\varepsilon}{2}}
$$

and

$$
\left\|t^{\frac{1}{2}} \theta(t)-\eta t^{\frac{1}{2}} G(t)\right\|_{L^{2}} \leq C t^{-\frac{\varepsilon}{2}}
$$

Proof. Well-known results for the heat equation or the Fokker-Planck equation [13] imply that

$$
\left\|S(\tau) \theta_{0}-\eta g\right\|_{L^{1}} \leq C e^{-\tau}
$$

when $\theta_{0} \in L^{1}$ and the second moment of the initial data is bounded. Moreover, since $m>3$ we also have [13] that

$$
\left\|t^{\frac{1}{2}} G(t) \theta_{0}-t^{\frac{1}{2}} \eta G(t)\right\|_{L^{2}} \leq C t^{-\frac{1}{2}}
$$

when $\theta_{0} \in L^{2}(m)$. Now, the proof follows from (3.1) together with Theorem 4.1.

Let us finally remark that since the dynamical system in self-similar variables becomes nonautonomous, we cannot apply the arguments based on La Salle principle that allows to restrict to signed initial data in [15, Lemma 3.3]. Nevertheless, the estimates obtained in sections 2 and 3 are enough to get the asymptotic simplification of the flow and its convergence to the linear behavior. However, the description of the asymptotic states of the solutions in the homogeneous space $L^{2}\left(\mathbb{R}^{2}\right)$ is still lacking, see [20] for a result in this direction for the forced periodic case. On the other hand, the estimates obtained in 
sections 2 and 3 show that the trajectories of the flow are compact both in $L^{2}(m)$ and $L^{1}$ by adapting to this case the arguments in [15, Lemma 2.2 and Lemma 2.5]. Let us finally mention that the restriction to the case $\gamma=1$ is mainly due to the use of the entropy functional and we believe much of the other information remains equally valid. All these questions will be further analyzed elsewhere.

Acknowledgements. JAC acknowledges support from DGI-MEC (Spain) project MTM 2005-08024. LCFF acknowledges support from the external postdoctoral program of CAPES (Brazil). We thank T. Gallay for several comments and discussions that improved the first version of this paper.

\section{References}

[1] A. Arnold, J. A. Carrillo, L. Desvillettes, J. Dolbeault, A. Jüngel, C. Lederman, P. A. Markowich, C. Villani, and G. Toscani, Entropies and equilibria of many-particle systems: an essay of recent research, Monatsh. Math. 142 (2004), 35-43.

[2] M. Cannone and G. Karch, Smooth or singular solutions to the Navier-Stokes system?, J. Diff. Eq. 197 (2004), 247-274.

[3] J. A. Carrillo and K. Fellner, Long time asymptotics via entropy methods for diffusion dominated equations, Asymptot. Anal. 42 (2005), 29-54.

[4] J. A. Carrillo and L. C. F. Ferreira, Self similar solutions and large time asymptotics for the dissipative quasi-geostrophic equations, preprint UAB, 2005.

[5] J. A. Carrillo and G. Toscani, Exponential convergence toward equilibrium for homogeneous Fokker-Planck-type equations, Math. Meth. Appl. Sci. 21 (1998), 1269-1286.

[6] J. A. Carrillo and G. Toscani, Asymptotic $L^{1}$-decay of solutions of the porous medium equation to self-similarity, Indiana Univ. Math J. 49 (2000), pp.113-141.

[7] E. A. Carlen and M. Loss, Optimal smoothing and decay estimates for viscously damped conservation laws with applications to the 2-D Navier-Stokes equations, Duke Math. J. 81 (1996), 135-157.

[8] D. Chae and J. Lee, Global well-posedness in the super-critical dissipative quasi-geostrophic equations, Comm. Math. Phys. 233 (2003), 297-311.

[9] P. Constantin, A. Majda and E. Tabak, Formation of strong fronts in the 2D quasi-geostrophic thermal active scalar, Nonlinearity 7 (1994), 1459-1533.

[10] P. Constantin, D. Córdoba and J. Wu, On the critical dissipative quasi-geostrophic equation, Indiana Univ. Math. J. 50 (2001), 97-107.

[11] P. Constantin and J. Wu, Behavior of solutions of $2 D$ quasi-geostrophic equations, SIAM J. Math. Anal. 30 (1999), 937-948.

[12] A. Córdoba and D. Córdoba, A maximum principle applied to quasi-geostrophic equations, Comm. Math. Phys. 249 (2004), 511-528.

[13] J. Duoandikoetxea and E. Zuazua, Moments, masses de Dirac et décomposition de fonctions, C. R. Acad. Sci. Paris Sér. I Math. 315 (1992), 693-698.

[14] M. Escobedo and E. Zuazua, Large time behaviour for convection-diffusion equations in $\mathbb{R}^{n}$, J. Funct. Anal. 100 (1991), 119-161.

[15] T. Gallay and C. E. Wayne, Global stability of vortex solutions of the two-dimensional Navier-Stokes equation, Comm. Math. Phys. 255 (2005), 97-129. 
[16] T. Gallay and C. E. Wayne, Invariants manifolds and the long-time asymptotics of the Navier-Stokes and vorticity equations on $\mathbb{R}^{2}$, Arch. Rat. Mech. Anal. 163 (2002), 209-258.

[17] J. García-Cuerva and J. L. Rubio de Francia, Weighted Norm Inequalities and Related Topics, North-Holland Mathematics Studies 116, 1985.

[18] L. Gross, Logarithmic Sobolev inequalities, Amer. J. Math. 97 (1975), 1061-1083.

[19] N. Ju, Existence and uniqueness of the solution to the dissipative 2D quasi-geostrophic equations in the Sobolev space, Comm. Math. Phys. 251 (2004), 365-376.

[20] N. Ju, The maximum principle and the global attractor for $2 D$ dissipative quasi-geostrophic equations, Comm. Math. Phys. 255 (2005), 161-181.

[21] T. Kato, The Navier-Stokes equations for an incompressible fluid in $\mathbb{R}^{2}$ with a measure as the initial vorticity, Diff. Int. Eq. 7 (1994), 949-966.

[22] T. Kato, Strong $L^{p}$ solutions of the Navier-Stokes equations in the $\mathbb{R}^{m}$ with applications to weak solutions, Math. Z. 187 (1984), 471-480.

[23] T. Kato, Strong solutions of the Navier-Stokes equations in Morrey spaces, Bol. Soc. Bras. Mat. 22 (1992), 127-155.

[24] P. G. Lemarié-Rieusset, Recent Developments in the Navier-Stokes Problem, Chapman \& Hall/ CRC Press, Boca Raton, 2002.

[25] J. Pedlosky, Geophysical Fluid Dynamics, Springer-Verlag, New York, 1987.

[26] S. Resnick, Dynamical Problems in Non-Linear Advective Partial Differential Equations, PhD thesis, University of Chicago, 1995.

[27] M. E. Schonbek and T. P. Schonbek, Asymptotic behavior to dissipative quasi-geostrophic flows, SIAM J. Math. Anal. 35 (2003), 357-375.

[28] M. E. Schonbek and T. P. Schonbek, Moments and lower bounds in the far-field of solutions to quasi-geostrophic flows, Discrete Contin. Dyn. Syst. 13 (2005), 1277-1304.

[29] E. M. Stein and G. Weiss, Introduction to Fourier Analysis on Euclidean Spaces, Princeton University Press, Princeton, N.J., 1971.

[30] G. Toscani, Kinetic approach to the asymptotic behaviour of the solution to diffusion equation, Rend. Mat. Appl. 16 (1996), 329-346.

[31] G. Toscani, Sur l'inégalité logarithmique de Sobolev, C. R. Acad. Sci. Paris Sér. I Math. 324 (1997), 689-694.

[32] J. Wu, Quasi-geostrophic-type equations with initial data in Morrey spaces, Nonlinearity 10 (1997) 1409-1420.

[33] J. Wu, Dissipative quasi-geostrophic equations with $L^{p}$ data, Electron. J. Diff. Eq. 56 (2001).

[34] J. Wu, The 2D dissipative quasi-geostrophic equation, Appl. Math. Lett. 15 (2002), 925-930. 
